\title{
CBCT Guided Endodontic Management of A Mandibular First Molar With Radix Entomolaris: A Case Report
}

\author{
Dr. Deepashree Paul ${ }^{1}$, Dr.Amitava Bora ${ }^{2}$, Dr.Piyush Singh $^{3}$, Dr.Gopal Chandra \\ Bera $^{4}$, Dr.Pratik Kumar Lahiri ${ }^{5}$ (Reader), Dr.Gautam Kumar Kundu ${ }^{6}$ (Professor \\ And Head Of The Department). \\ ${ }^{1,3,4,5,6}$ Department of Pedodontics and Preventive Dentistry, Guru Nanak Institute Of Dental Science And \\ Research, Kolkata, India \\ ${ }^{1,3,4}$ Final Year MDS, Post Graduate Trainees \\ ${ }^{2}$ Clinical Tutor, Department of Dentistry, Calcutta Medical College \& Hospital, Kolkata, India
}

\begin{abstract}
Knowledge about the possible existence and the racial prevalence is mandatory for successful endodontic treatment of cases ofRadix Entomolaris (RE). Factors such as an extra cusp usually buccal (protostylid), prominent distolingual lobe, complexity of the external contour of the furcation and convexity in the cervical region help to diagnose the presence of an RE. Radiographically, double periodontal ligament images or outline of the distal root contour can hint the presence of an RE. An angled radiograph $\left(25-30^{\circ}\right) \mathrm{can}$ be more useful in this regard. Three-dimensional imaging techniques based on computed tomography (CT) and cone beam computed tomography (CBCT) have been able to predict the exact morphology of an RE noninvasively. This is a case report of a successful endodontic treatment of an 11 years old female patient with bilateral radix entomolaris.
\end{abstract}

Keywords: Bilateral Radix Entomolaris, endodontic treatment, Cone beam computed tomography.

\section{Introduction}

The success of any endodontic treatment has always been dictated by the comprehensive knowledge and the clinical expertise of the dental surgeon. Thus, it is essential to be oriented with the possible morphological variations. Barett once quoted "of all the phases of study of the human anatomy one of the most complex is that of pulp cavity morphology."A major variant from the two-rooted morphology of a mandibular first molar is the occurrence of a supernumerary root. Radix entomolaris (RE) is the presence of an additional distolingual root, which was first mentioned in literature by Carabelli[1] in 1844. Radix paramolaris(RP) is known as the additional mesiobuccal[2]root which was first described by Bolk in 1915[3]. Incidence of RE has been documented as $5.97 \%$ in the Indian population and, $3 \%$ in the Asian population [4].

Identification and treatment of an RE is important because a missed canal becomes a nidus for infection and can compromise the treatment results. Factors such as an extra cusp usually buccal (protostylid), prominent distolingual lobe, complexity of the external contour of the furcation and convexity in the cervical region help to clinically diagnose the presence of an RE.

Clinical suspicion of an extra root should always be corroborated radiographically. Radiographically, exact diagnosis of root morphology is a challenge in RE and RP cases. Double periodontal ligament images or outline of the distal root contour can hint the presence of a RE, likewise double periodontal ligament images or outline of the mesial root contour can hint the presence of a RP.

Accurate diagnosis of a complex three dimensional tooth morphology cannot be achieved with conventional two dimensional Intra-oral periapical radiographs or orthopantomogram (OPG). Multiple Intra-oral periapical radiographs in different angulations are required to diagnose complex morphologies of three dimensional teeth. The limitations of conventional two dimensional radiographs can be overcome with three dimensional Imaging like Computed Tomography (CT), DentaScan, cone-beam computed tomography (CBCT) etc. Advantages of CBCT include lower radiation dosage than helical CT, Compact equipment design, superior images to Panoramic, Lower cost and Lower heat load. The short comings include Metal artefacts, worse low contrast detectability, longer scan time,motion artefacts, slightly inferior quality to conventional CT. Weighing the pros and cons CBCT has been found to be apt for accurate diagnosis in these cases.

During endodontic management of RE or RP access cavity should be properly modified to trapezoidal or oval form rather than the conventional triangular form for ease of approach of the extra root during biomechanical preparation. Use of magnification devices (operative microscope, magnification loop) are also advocated during treatment. 


\section{Case Report}

An eleven year old female patient reported to the department of Pedodontics and Preventive Dentistry of the institute with the chief complaint of pain in the lower left back tooth region since the past few days. History of present illness revealed the pain was Spontaneous, moderate in intensity and throbbing in nature. Pain increased at night or in supine posture. Patient's past medical and dental history was non-contributory. On inspection 36 was grossly carious and tender on vertical Percussion. No abnormality was detected on Palpation and periodontal probing was within the physiological limits. A heat test resulted in severe lingering pain. The involved tooth showed a premature response to electrical pulp stimulation. The Intra oral periapical radiograph (IOPAR) revealed radiolucency involving the mesial pulp horn with widening of the periodontal ligament space. A broader than normal distal root that appeared to have an open apex was seen (fig 1). Based on these findings, the Provisional diagnosis was Dental caries with Irreversible pulpitis in 36.

Considering the age of the patient and extent of apical closure a clinical decision of performing Apexification of 36, followed by Stainless Steel Crown was taken.

On the first visit access cavity was prepared under Local anaesthesia. The pulp tissue was extirpated using barbed broaches, $1.5 \%$ Sod. Hypochlorite was used for irrigation, and no. 10 and $15 \mathrm{~K}$-files were placed in the canals to obtain the working lengths by Ingle's method. During which a possibility of a Radix Entomolaris was felt. Clinically there weren't any open apices as expected from the IOPAR.

Patient was adviced for a Cone Beam Computed Tomography, The transverse, axial, and sagittal CBCT sections of whole mandibular arch were taken. The CBCT Axial section confirmed the presence of Bilateral Radix Entomolaris of 36 and 46 (fig 2). It was identified as Carlsen's Type A Radix Entomolaris with 36 (fig 3).

At the second visit, the access cavity was modified into a trapezoidal form to locate the disto-lingually located RE easily. A clinical examination was carried out with a DG16 endodontic explorer (Hu-Friedy, Chicago, IL, USA), and examination with magnification loop revealed two mesial and two distal canal orifices. An apex locator was used to confirm the working lengths of each canal using PropexPixi by Dentsply, Maillefer, Switzerland. Bio-mechanical preparation was performed by rotary instrumentation using nickeltitaniumProtaper System (Densply, Maillefer, Switzerland). Canals were enlarged upto size F2 along with repeated irrigation with normal saline and $1.5 \%$ sod. hypochlorite. Canals were dried and Master cones were placed and confirmed using RadioVisioGraphy. Obturation was done with Protaper gutta-percha cones (Dentsply India) and AH Plus resin sealer (Dentsply, India)(fig 4). The access cavity was then restored with posterior composite resin. Stainless steel crown (3M) was cemented (fig 5) and patient has been kept on followup since past two and a half years.

\section{Discussion}

The exact cause of radix entomolaris is still uncertain. It is believed to arise from any disturbance during odontogenesis or may be due to penetration of an atavistic gene (recurrence of a trait from ancestral character, usually due to genetic recombination). Incidence varies from 3 to $30 \%$ and also among different populations. De Moor et al [12] had classified RE based on the curvature in the bucco-lingual direction. Type I refers to straight root / canals, Type II refers to a curvature at the orifice opening and Type III refers to RE with two curvatures, one at the coronal level and the other at the middle third. Carlsen and Alexandersen described four different types of RE according to the location of the cervical part of the RE which allows identification of separate and non-separate RE[13]. Type A and Type B refer to a distally located cervical part of the RE with both normal and oneabnormal distal root components, respectively. Type $\mathrm{C}$ with a mesially located cervical part and Type AC with a central location, between the distal and mesial root components[14]. This was a case of Carlsen's Type A Radix Entomolaris.

To achieve a correct diagnosis minimum of two diagnostic radiographs are necessary using buccal object rule. Additional cusps are an indication of the possibility of Radix Entomolaris, usually an extra buccal cusp (protostylid).

By using extracted teeth, Walker and Quackenbush[5] found a prevalence of $14.6 \%$ in Hong Kong Chinese and Loh[6] found a prevalence of $7.9 \%$ in Chinese subjects of Singapore. A frequency of $3 \%$ is documented in the African population[7] while in Indian and Eurasian populations the frequency is less than 5\%[8]. Interestingly, it has been considered as Eumorphic(normal morphological variation) among mongoloids and dysmorphic (unusual) in Caucasians due to the varying prevalence rates.

Advanced diagnostic aids help in the better identification and visualization of all the canals.Dose levels for CBCT imaging are far below those of clinical MSCT protocols[9]. As it is essential to optimise the radiation dose while achieving a sufficient image quality, $\mathrm{CBCT}$ has been found to be an apt 3D imaging technique to understand the 3D morphology of the tooth in detail at a justified exposure to radiation. 
The problems commonly encountered during the treatment of Radix Entomolaris are difficulty in Radiographic interpretation, inability to locate the extra canal due to unexpected morphology, modification of the access cavity and confusion in working length determination due to super imposition of canals in two dimensional radiographic methods. Apart from these difficulties clinicians are prone to commit some iatrogenic errors like straightening of a root canal resulting in error in working length,zipping,transportation, ledge formation or even perforation. Access cavity modification should be done following the dentinal map. For that reason access cavity should be properly modified to trapezoidal or oval form rather than the conventional triangular form for ease of approach of the extra root during biomechanical preparation. Use of magnification devices (operative microscope, magnification loop) are also advocated during treatment.

Bilateral occurrence of the RE ranges from 50 to 67\%[10]. A recent study reported $60 \%$ occurrence of bilateral radix Entomolaris cases amongst total radix entomolaris cases, in an Indian population, though the overall occurrence was less $(2.19 \%)$ [11]

\section{Conclusion}

What the mind does not know the eyes cannot see!'Knowledge regarding the possible existence and the factors and prevalence of Radix Entomolaris helps deal with the unexpected.Three-dimensional imaging techniques based on cone beam computed tomography (CBCT) for visualizing the true morphology non-invasively is a key to successful endodontic treatment of a case of Radix Entomolaris.In case of unavailability of CBCT, multiple radiographs in varying angulations have proved to be useful.

\section{FIGURES}

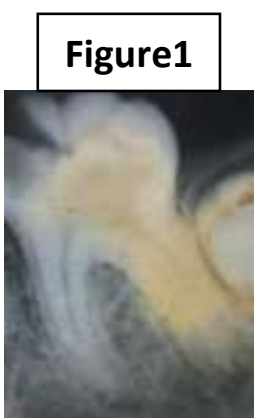

Figure 2

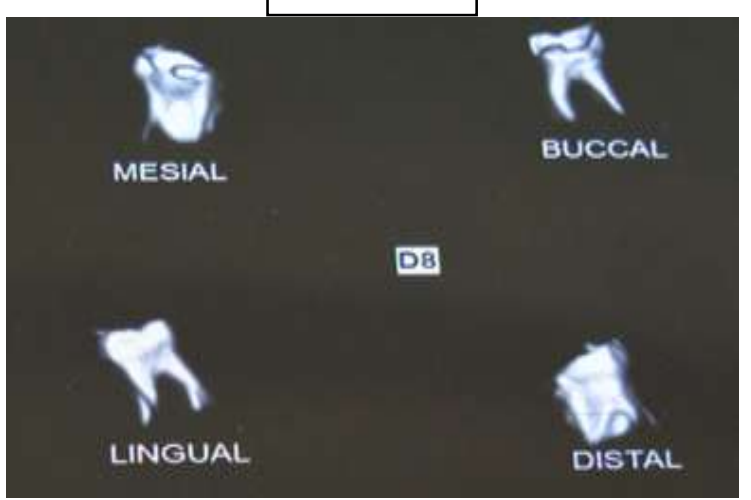



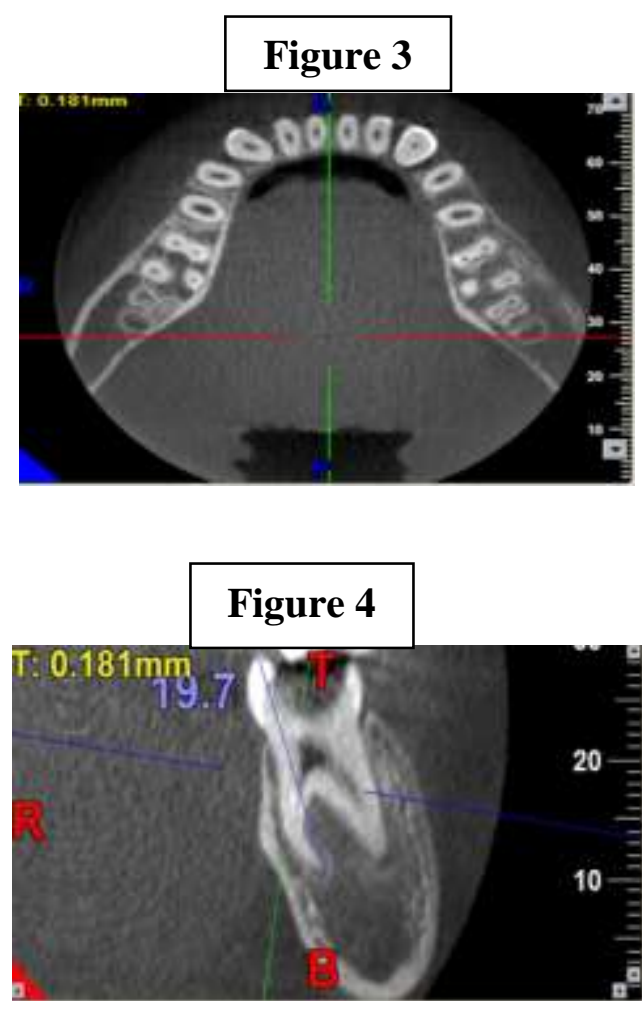

\section{Figure 5}

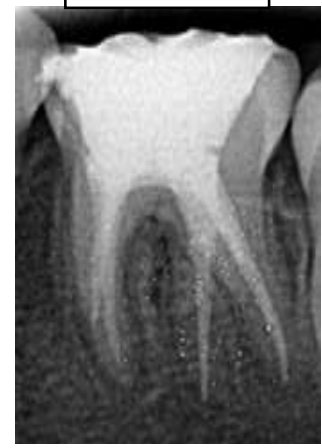

Figure 6

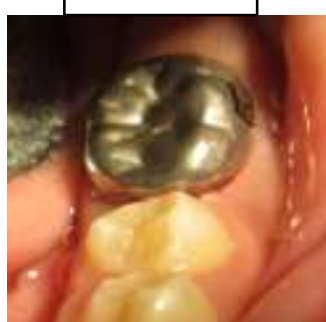




\begin{tabular}{|ll|}
\hline \multicolumn{2}{|l|}{ Legends: } \\
1. & Pre-operative intraoral periapical radiograph \\
2. & 3 D view of 36 (tooth of interest) \\
3. & Axial view CBCT of Mandible \\
4. & Coronal view CBCT of Distal roots \\
5. & Post obturation Radiovisuography image \\
6. & Stainless steel crown cemented
\end{tabular}

\section{References}

[1]. Carabelli G. SystematischesHandbuch der Zahnheikunde. 2 nd ed. Vol. 114. Vienna: Braumuller and Seidel; 1844.

[2]. Carlsen O, Alexandersen V. Radix paramolaris in permanent mandibular molars: Identification and morphology. Scand J Dent Res 1991;99:189-95

[3]. Bolk L. Bemerkungen u berWurzelvariationen am menschlichenunterenMolaren. Zeiting fur Morphologie Anthropologie $1915 ; 17 ; 605-10$.

[4]. Garg AK, Tewari RK, Kumar A, Hashmi SH, Agrawal N, Mishra SK. Prevalence of Three-rooted Mandibular Permanent First Molars among the Indian Population. Journal of Endodontics 2010;36(8):1302-5.

[5]. Walker RT, Quackenbush LE. Three-root lower first permanent molar in Hong-Kong Chinese. Br Dent J 1985;159:298-9. Back to cited text no. 22

[6]. Loh HS. Incidence and features of three-rooted permanent mandibular molars. Aust Dent J 1990;35:437-7.

[7]. Sperber GH, Moreau JL. Study of the number of roots and canals in Senegalese first permanent mandibular molars. IntEndod J 1998;31:112-6.

[8]. Tratman EK. Three-rooted lower molars in man and their racial distribution. Br Dent J 1938;64:264-74.

[9]. M. Loubele et al. / European Journal of Radiology xxx (2008) xxx-xxx. (Article in press)

[10]. Calberson FL, De Moor RJ, Deroose CA. The radix entomolaris and paramolaris: Clinical approach in endodontics. J Endod 2007;33:58-63

[11]. Bharti R, Arya D, SaumyendraVS ,Kulwinder KW, Tikku AP, Chandra A. Prevalence of Radix Entomolaris in an Indian Population. Indian J Stomatol 2011; 2(3):165-67.

[12]. Demoor, Deroose, Calberson. The Radix Entomolaris inmandibular first molars : an endodontic challenge IntEndod J 2004 ; 37:789-799

[13]. Calberson FL, De Moor RJ, DerooseCA.The radix entomolaris and paramolaris:clinical approach in endodontics. Journal ofEndodontics2007;33(1):58-63.

[14]. Carlsen O, Alexandersen V. Radixparamolaris in permanent mandibularmolars: identification and morphology.European Journal of Oral Sciences 1991; 99(3): 189-95 